\section{Dagmar Wemmer}

Von März 2009 bis Mai 2010 wurde ein Pilotprojekt zur Integration eines phytotherapeutischen Therapieangebots für Patientinnen und Patienten einer medizinischen Akutabteilung am Spital in Wattwil durchgeführt. Diskussionen in Parlamenten und Fachkreisen, die Komplementärmedizin wieder als Leistung in die Grundversicherung aufzunehmen, die hohe Nachfrage nach komplementärmedizinischen Angeboten durch die Bevölkerung und der naturnahe Standort des Spitals (Wattwil/Toggenburg) lieferten die Motivation für die Unternehmung.

Die Fragestellung lautete, ob die Einführung von phytotherapeutischen pflegerischen Anwendungen und die Verordnung von pflanzlichen Arzneimitteln auf einer medizinischen Akutstation möglich, sinnvoll und nutzbringend sind. Bei den pflegerischen Anwendungen wurden überwiegend Zubereitungen (Tinkturen, Arzneitees, ätherische Öle) verwendet, die aus Drogen hergestellt wurden, von denen anerkannte Monographien zur Qualität (Europäische und Schweizer Pharmakopöe) und/ oder Wirksamkeit (ESCOP, Kommission E) vorliegen. Die eingesetzten Spezialitäten waren alle von Swissmedic, dem Schweizerischen Heilmittelinstitut, zugelassen.

*Dr. med. Dagmar Wemmer, Ärztin für Allgemeinmedizin und Naturheilverfahren, war mit der Projektleitung beauftragt. Das Projekt wurde in der Spitalregion Fürstenland-Toggenburg (SRFT) in der medizinischen Akutabteilung des Spitals Wattwil durchgeführt.

\section{Pilotprojekt zur Integration von Phytotherapie in der Akutmedizin*}

Folgende Hypothesen wurden für die Evaluation formuliert:

- die Anwendung von Phytotherapie stellt eine Erweiterung des Therapieangebots dar,

- konventionelle Medizin und Phytotherapie ergänzen sich,

- es ergibt sich ein Mehrwert für Patienten, Leistungserbringer und Trägerschaft,

- das Angebot erfordert keine zusätzlichen Personalressourcen.

\section{Durchführung}

Nach der Bestimmung der Abteilung, die für das Projekt infrage kam (ausgewählt wurde die medizinische Akutabteilung mit 22 Betten), der Bildung eines interdisziplinären Projektkernteams und der Einrichtung eines separaten Raums für die
Vorbereitung von phytotherapeutischen Anwendungen (Wickel, Auflagen, Einreibungen, Fussbäder, Tees) und der Einlagerung der pflanzlichen Arzneimittel, Zubereitungen und Drogen wurden zwischen Ende Mai und Anfang August 2009 Ärzte und Pflegende von der Projektleitung in den vorher ausgewählten Anwendungen bzw. den zur Verfügung stehenden Spezialitäten geschult. Die Schulung für die Pflegenden umfasste 11 Nachmittage mit Theorie und Praxis der Phytotherapie. Die Ärzte wurden an 3 Nachmittagen mit den Grundlagen der Phytotherapie vertraut gemacht sowie in der Verordnung ausgewählter pflanzlicher Arzneimittel (ausschliesslich von Swissmedic zugelassene Spezialitäten) geschult. Details zur Schulung werden in Tabelle 1 und 2 präsentiert.
Tab. 1. Überblick über die Inhalte der pflegerischen Module

Modul 1: Einführung (Überblick, Begriffsklärungen, Forschung, Rechtliches,

Material für die pflegerischen Anwendungen, Geschichte der Heilpflanzen)

Modul 2: Wirkstoffe in Arzneipflanzen

Modul 3: Anwendungsmöglichkeiten von Arzneipflanzen, Wickellehre, Teekunde

Modul 4: Phytotherapeutische Anwendungen bei Hauterkrankungen und Verletzungen

Modul 5: Phytotherapeutische Anwendungen bei Erkrankungen der Psyche, des Nervensystems und

bei allgemeinen Befindlichkeitsstörungen

Modul 6a: Phytotherapeutische Anwendungen bei Störungen des Verdauungssystems I

Modul 6b: Phytotherapeutische Anwendungen bei Störungen des Verdauungssystems II

Modul 7: Phytotherapeutische Anwendungen bei Störungen des Herz-Kreislauf-Systems

Modul 8: Phytotherapeutische Anwendungen bei Nieren- und Blasenerkrankungen

Modul 9: Phytotherapeutische Anwendungen bei Atemwegserkrankungen und zur Stärkung des Immunsystems

Modul 10: Phytotherapeutische Anwendungen bei Erkrankungen des muskulo-skelettalen Systems

Modul 11: Phytotherapeutische Anwendungen bei Schmerzen

Modul 12: Phytotherapeutische Anwendungen in der Palliativmedizin

Arzneipflanzenexkursion

Freiwillige theoretische und praktische Prüfung

${ }^{a}$ Ein von der SMGP überprüftes Skript zu den Modulen 1-12 stellt die Autorin gegen ein Entgelt zur Verfügung.

Tab. 2. Inhaltlicher Überblick der Ärzteschulung

Was ist Phytotherapie? (Abgrenzungen, Begriffsklärung, Forschungsstand)

Portraits der für die Akutmedizin vorgesehenen Arzneipflanzen/Spezialitäten gemäss eigens erstellter Liste

Pflegerische Anwendungen im Rahmen der Phytotherapie

Arzneipflanzenexkursion

\section{KARGER}

Fax +497614520714 Information@Karger.de www.karger.com
(๑) 2012 S. Karger GmbH, Freiburg
Dr. med. Dagmar Wemme

Aufeld 9, 9607 Mosnang, Schweiz

dagslys@web.de

www.phytopraxis.ch 
Das Pflegepersonal wurde in folgenden praktischen phytotherapeutischen Anwendungen instruiert und geschult:

\section{Waschungen}

Malventeewaschung, Pfefferminzteewaschung, Rosmarinwaschung, Salbeiteewaschung, Schwarzteewaschung.

\section{Kompressen/Auflagen}

Arnikaauflage, Arnika-Herzkompresse, Calendulaauflage, Eukalyptusölauflage, Johanniskrautölkompresse, Johanniskrautölauflage, Kamillenteedelölkompresse, Lavendelölauflage, Leinsamenkompresse, Malventeeauflage, Melissenölkompresse, Meerrettichkompresse, Quarkauflage, Schwarzteekompresse, Senfauflage, Spitzwegerichauflage, Thymianölkompresse, Thymianteeauflage, Zwiebelauflage.

\section{Wickel}

Quark-Halswickel, Zitronenwickel, feuchtwarmer Kamillenölwickel, Schafgarbenwickel.

\section{Einreibungen}

Lavendelöl-Fusseinreibung, Lavendelöl-Handeinreibung, Kümmelöl-Baucheinreibung, Rosmarinöl-Fusseinreibung, Rosmarinöl-Handeinreibung.

\section{Fussbäder}

Heublumenfussbad, Kamillenfussbad, Lavendelfussbad, Rosmarinfussbad, Salbeifussbad, Senfmehlfussbad.

\section{Arzneitees}

Teemischungen: Schlaftee in Beuteln; Blasen-Nieren-Tee, Brust- und Husten-Tee, Erkältungstee, Magentee, kompresse, Kamillenölauflage, Laven-

Rheumatee (alle Mischungen in Beuteln, bei Swissmedic zugelassene Arzneimittel von Sidroga AG, Zofingen, Schweiz).

Einzeltee: Fencheltee, Kamillentee, Lavendelblütentee, Malventee (Malva silvestris), Melissentee, Orangenblütentee, Pfefferminztee, Rosmarintee, Salbeitee, Schafgarbentee, Thymiantee, Wermuttee (die Einzeldrogen wurden von der Kantonsapotheke St. Gallen bezogen).

\section{Evaluationsbögen und Prüfung}

Beide Berufsgruppen erhielten Evaluationsbögen, die bei jeder phytotherapeutischen Anwendung/Verordnung auszufüllen waren und sowohl subjektive Aussagen der Patienten als auch objektive Beobachtungen, Dauer und Grund der Anwendung dokumentierten. Der Evaluationsbogen wurde einfach gehalten, enthielt jedoch die nötigen Rubriken (Abb. 1).

Nachdem sie einige Erfahrungen gesammelt hatten, wurde den Pflegenden die Möglichkeit gegeben, eine freiwillige theoretische und praktische Prüfung abzulegen, die im November 2009 unter Vorsitz der Projektleitung und einer wissenschaftlichen Mitarbeiterin der Schweizerischen Medizinischen Gesellschaft für Phytotherapie (SMGP) stattfand.

Der Prüfungsinhalt gliederte sich in einen inhaltlichen Teil (Darstellung eines Arzneipflanzenportraits nach bestimmten Kriterien wie Beschreibung, Vorkommen, Droge, Inhaltsstoffe, Historisches, Forschung, Wirkungsweise, Indikationen, Kontraindikationen, Nebenwirkungen, Anwendungsweisen, Besonderheiten)

Evaluation Phytopharmaka

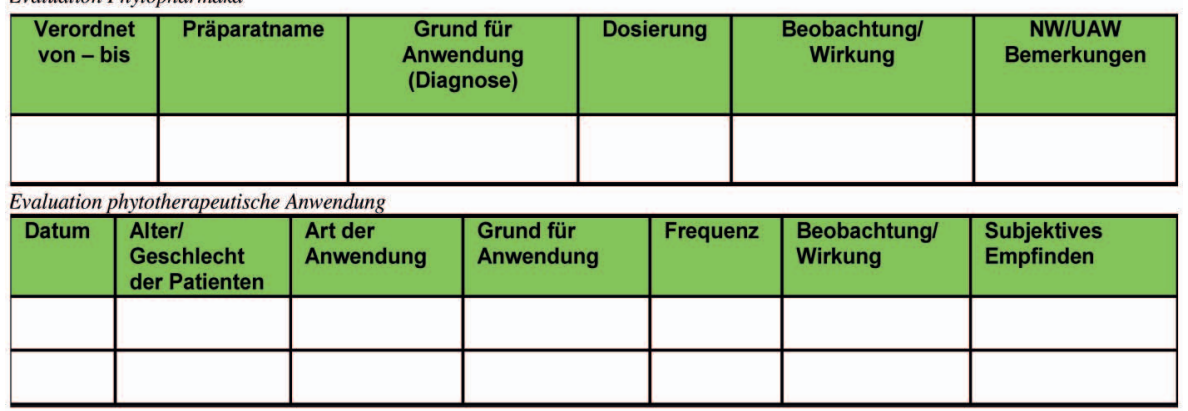

Abb. 1. Evaluation der Phytopharmaka sowie der phytotherapeutischen Anwendungen. sowie einen praktischen Teil, in dem entweder eine phytotherapeutische Anwendung (mit Indikation, Vorbereitung, Information des Patienten, Durchführung, Besonderheiten und Nachsorge) direkt am Patienten angewendet oder anhand eines Fallbeispiels erklärt werden musste. Die 6 zur Prüfung angemeldeten Pflegefachfrauen bestanden diese erfolgreich. Diese Qualifizierung soll es den Pflegenden ermöglichen, neue Mitarbeitende in der Durchführung phytotherapeutischer Anwendungen anzuleiten.

\section{Abschlussevaluation/Ergebnisse}

Die Abschlussevaluation ergab nach einem Anwendungszeitraum von 10 Monaten folgende Ergebnisse:

\section{Evaluation der pflegerischen} phytotherapeutischen Anwendungen Wickel und Auflagen wurden am häufigsten angewandt, dicht gefolgt von Arzneitees. Dies ist verständlich, da Tees die einfachste Anwendungsart darstellten - mit geringer Vorbereitungszeit und vielfältigen Indikationen. Wickel und Auflagen waren als breite Anwendungsmöglichkeit mit zahlreichen Indikationen ebenfalls gegeben, während Fussbäder und Waschungen wenigen, spezifischen

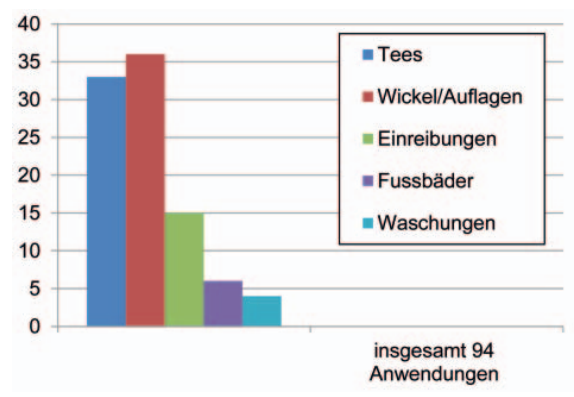

Abb. 2. Anzahl Anwendungen pro Anwendungsart (5 verschiedene, wobei Wickel und Auflagen zusammen erfasst wurden). Anwendungszeitraum 10 Monate, ausser für die Tees, die erst ab der fünften Woche der Projektlaufzeit erfasst wurden. Hochgerechnet ergeben sich daraus die meisten Anwendungen (37 gegenüber 36 bei Wickeln/Auflagen); daher stehen sie an erster Stelle. 
Abb. 3. Häufigkeit der Behandlungen in Relation zur Behandlungsdauer.

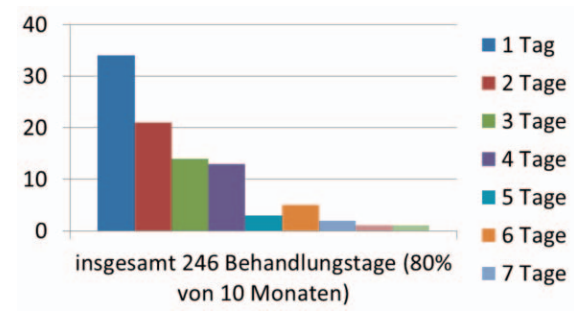

Abb. 4. Darstellung der Frequenz der Anwendungen pro Pflanze (hellblaue Säule, jeweils ganz rechts) sowie der Häufigkeit der verschiedenen externen Anwendungsarten (verschiedene Säulenfarben) pro Pflanze. Mit Lavendel wurde demnach keine Waschung durchgeführt, hingegen kamen die übrigen Anwendungen - je 4-mal Einreibung und Wickel, 2-mal Fussbad an insgesamt 28 Behandlungstagen - zum Einsatz. Die Zitrone hingegen wurde einzig als Wickel nur 2-mal an insgesamt 3 Behandlungstagen angewendet.

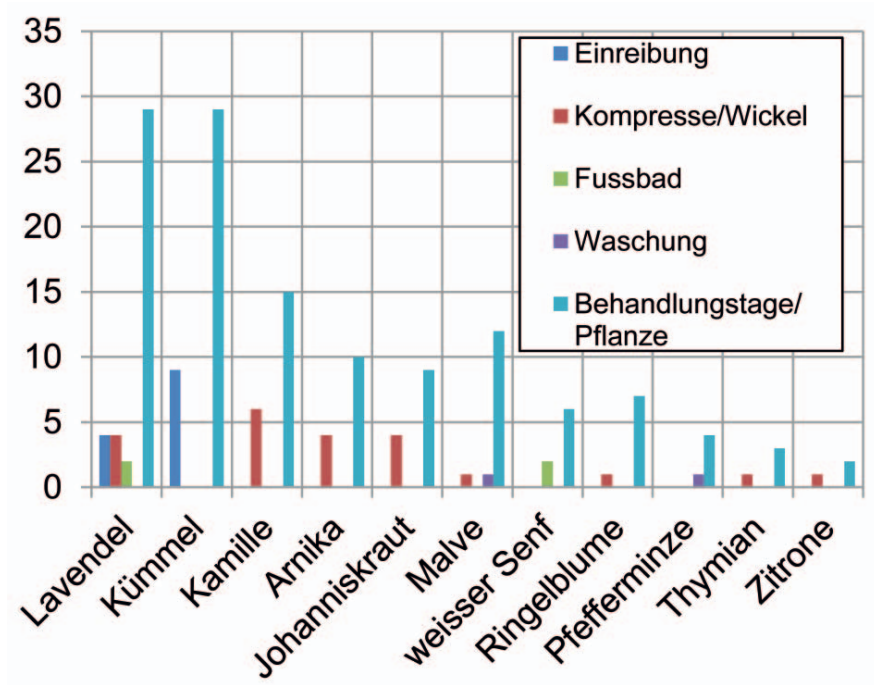

Unruhe und Ängste verursachte Einschlaf- und Durchschlafstörungen.

- Zystitiden.

Die Evaluationsdauer für die Tees fällt einen Monat kürzer aus, da deren Einbezug in die Evaluation erst nach der Startphase des Projektes beschlossen wurde.

Aus den Evaluationsbögen ergaben sich bezogen auf sämtliche phytotherapeutischen Anwendungen folgende allgemeine Befunde:

- $100 \%$ positive Rückmeldungen (subjektive Aussagen von Patientinnen und Patienten, dokumentiert vom Pflegepersonal).

- 3,2\% unangenehme Begleiterscheinungen (3 von 94 Fällen), ohne dass die Anwendung abgebrochen werden musste (subjektive Aussagen von Patientinnen und Patienten, dokumentiert vom Pflegepersonal).

- Objektiv 56\% beobachtbare Wirkungen (in 53 von 94 Fällen fachlich beobachtet, erhoben und dokumentiert vom Pflegepersonal).

Das Ergebnis der vom Pflegepersonal dokumentierten Patientenrückmeldungen zeigt, dass sämtliche phytotherapeutischen Anwendungen eine hohe Akzeptanz bei den Patientinnen und Patienten erreichen konnten und zwar durchgehend auf allen Anwendungsebenen (Tee, Wickel, Fussbad, Einreibung, Kompresse, Waschung). Die objektiv beobacht- 


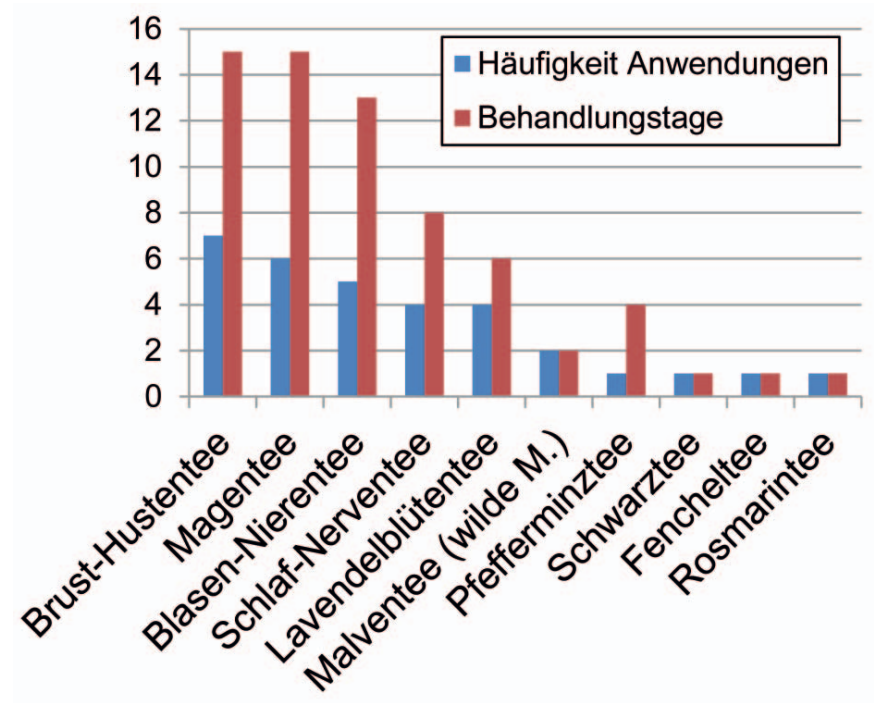

Abb. 5. Einsatz der verschiedenen Arzneitees in Relation zu den Behandlungstagen. Brustund Hustentee wurde bei 7 Patienten an 15 Behandlungstagen verabreicht, während Schwarz-, Fenchel- und Rosmarintee bei nur je einem Patienten an einem Behandlungstag verabreicht wurden. baren Wirkungen (jeweils ausschliesslich Besserungen) wären mit der Anwendung einer Visual-AnalogSkala (VAS) bei Schmerzzuständen vermutlich höher gewesen, jedoch wurden sie (abdominelle Schmerzen bei Obstipation, Schmerzen bei entzündlichen Prozessen wie Phlebitis, Erysipel, Podagra; Kopfschmerzen) über die subjektiven Äusserungen der Patienten evaluiert.

Insgesamt gab es nur drei negative Rückmeldungen (zwei zum Geschmack des Magentees, eine zu Hautbrennen am Ende einer Arnika-Auflage). Die Anwendungen mussten deshalb nicht abgebrochen werden. Da zahlreiche Patientinnen und Patienten den Wunsch hatten, die phytotherapeutische Anwendung nach Austritt zuhause weiterzuführen, verfasste die Projektleitung Informationsblätter $\mathrm{zu}$ einzelnen Anwendungen mit einer Beschreibung der Arzneipflanze und einer Anleitung zur Anwendung.

In den Projektteamsitzungen wurden Abweichungen von den vorgesehenen Anwendungen, die sich aus den Evaluationsbögen ergaben (z.B. wurde das Süssen des Magentees auf Wunsch einer Patientin entgegen den Empfehlungen erlaubt), besprochen und im weiteren Verlauf des Projektes korrigiert. Vonseiten des Pflegeteams wurden die phytotherapeutischen Anwendungen sinnvoll kombiniert.
Gleichzeitig wurden in den unabhängig vom Pilotprojekt stattfindenden 14-täglichen Fallbesprechungen der Pflege regelmässig die phytotherapeutischen Anwendungen mit einbezogen.

Eine personelle Aufstockung war im gesamten Zeitraum der Evaluation nicht erforderlich. Während der Schulungen des Pflegepersonals wurden jedoch Aushilfskräfte beigezogen.

\section{Evaluation der ärztlichen \\ Verordnungen von planzlichen}

Arzneimitteln

Vier Monate nach der Schulung in Phytotherapie lag kein ausgefüllter Evaluationsbogen der Ärzteschaft vor. Mittels Fragebogen an die betroffenen Ärzte wurde versucht, die Gründe für die Verordnungszurückhaltung zu erfassen. Es zeigte sich eine erhebliche Unsicherheit und Skepsis bezüglich der Anwendungsmöglichkeiten von pflanzlichen Arzneimitteln im Akutbereich. Als häufigste Gründe für die Unsicherheit wurden genannt: «Zu wenig Studien» und «zu kurze Liegezeit der Patienten». Hierbei muss ergänzt werden, dass ausschliesslich Neuverordnungen evaluiert werden sollten. Pflanzliche Arzneimittel, die bereits vor dem Spitalaufenthalt vom Hausarzt verordnet waren, wurden weiterhin eingesetzt, aber nicht im Evaluationsbogen erfasst.
Nach der Fragebogenauswertung wurde einerseits eine «Phyto-Hotline» installiert, die eine kurzfristige Beratung zur Verordnung von pflanzlichen Arzneimitteln gewährleisten sollte, und andererseits die Möglichkeit eines phytotherapeutischen Konsiliums erwogen und angeboten. Eine konsiliarische Begleitung der Chefarztvisite führte zu einem relativ hohen Zeitaufwand im Vergleich zur Beratungsmöglichkeit und wurde daher nicht fortgesetzt. Während des Projektes wechselte fast das gesamte Ärzteteam, sodass eine neue, vereinfachte und schematisierte Kurzschulung zur Verordnung einer überschaubaren Menge von pflanzlichen Arzneimitteln für die neu eingetretenen Mediziner durchgeführt wurde. Die oben genannte Unsicherheit und Skepsis blieb dennoch bestehen, sodass eine Evaluation der Anwendung von pflanzlichen Arzneimitteln schlussendlich unmöglich war.

\section{Schwierigkeiten und Hürden}

Aufgrund der kurzen Liegezeit der Patienten auf einer Akutstation liessen sich phytotherapeutische Anwendungen überwiegend nur in einem Zeitrahmen von 1-4 Tagen durchführen. Ein längerer Beobachtungs- und Beurteilungszeitraum hätte sich über den Spitalaufenthalt hinausziehen müssen, was aus logistischen Gründen nicht möglich war. Fluktuationen im Personalbereich, insbesondere bei der Ärzteschaft, erforderten mehrere repetitive Schulungen, um den Wissensstand aufrechtzuerhalten.

Die Struktur und die Aufgabenschwerpunkte des medizinischen Akutbereichs im Spital erforderten häufig ein Zurückstellen möglicher, aber aus Zeitgründen nicht durchführbarer, phytotherapeutischer Massnahmen zugunsten der notwendigen akutmedizinischen und pflegerischen Anforderungen. Mehrfach äusserten Pflegende, dass sie gerne phytotherapeutische Massnahmen angewendet hätten, die jeweilige Anwendung (Angebot beim Patien- 
ten, Vorbereitung der Massnahme, Anwendung und Überwachung, Nachbereitung, Ausfüllen des Evaluationsbogens) jedoch nicht in die engen Zeitfenster, insbesondere wenn Notfälle behandelt werden mussten, passten.

\section{Schlussfolgerungen und Diskussion}

Das Pilotprojekt «Integration von Phytotherapie in die Akutmedizin», das am 25. März 2009 am Spital Wattwil mit dem Projektteam startete und am 11. Mai 2010 in der Geschäftsleitungssitzung der Spitalregion Fürstenland-Toggenburg (SRFT) abgeschlossen wurde, zeigte durchwegs eine sehr hohe Patientenzufriedenheit und -akzeptanz. Bezüglich der Personalressourcen wurden lediglich Aushilfen während der Schulungszeit benötigt, jedoch wären mit einer geringen Aufstockung des Pflegepersonals mehr phytotherapeutische Anwendungen möglich gewesen. Die Motivation von Patientinnen und Patienten, phytotherapeutische Massnahmen kennenzulernen und gegebenenfalls zuhause auch eigenständig fortzuführen, ist hoch und zeigt, dass das Vertrauen in die Phytotherapie von Patientenseite ausgeprägt vorhanden ist und einem Behandlungsbedürfnis entspricht.

Der Einsatz pflanzlicher Arzneimittel ist dagegen auf einer Akutstation sehr begrenzt, da die Aufenthaltsdauer $\mathrm{zu}$ kurz ist, um eine Wirkungsentfaltung zu beobachten. Die multimorbiden Patienten haben zudem sehr oft bereits eine Vielfalt konventioneller Medikamente verordnet. Bei den Ärzten herrscht mangels praktischer Erfahrung eine grosse Unsicherheit im Umgang mit pflanzlichen Arzneimitteln. Das ergab sich aus der Zwischenbefragung und den Diskussionen anlässlich der letzten Projektteamsitzung. Die Phytotherapie wurde vonseiten der Ärzte nur wenig mitgetragen.
Nach Projektabschluss wurde in der SRFT diskutiert, in welchem Umfang in Zukunft die Phytotherapie in den Spitälern Wattwil und Wil Anwendung finden wird und welche Massnahmen für eine professionelle Umsetzung und Bewahrung bzw. Erweiterung der Kenntnisse in Phytotherapie getroffen werden müssen.

Es ist diskussionswürdig, ob Skepsis und Unsicherheit bei der Verordnung von pflanzlichen Arzneimitteln in der Ärzteschaft im Rahmen der Akutmedizin Ausdruck der Unmöglichkeit der Integration von Phytotherapie in die Akutmedizin oder eher Folge einer auch heute noch einseitig auf konventionelle Therapie ausgerichteten Hochschulausbildung ist. Die Vernachlässigung der Tatsache, dass wir erst seit einem verhältnismässig kurzen Zeitraum der Medizingeschichte über synthetische Arzneimittel und hochgereinigte Naturstoffe verfügen und der kranke Mensch Jahrtausende zuvor in allen Kulturen mit Arzneipflanzen behandelt wurde, sowie auch der Verlust des Bewusstseins, dass der Mensch Teil der Natur ist, lassen den Schluss $\mathrm{zu}$, dass im akademischen Bereich wichtige ergänzende Kenntnisse über die Wirksamkeit und die Anwendungsweisen von Arzneipflanzen trotz jahrzehntelanger Forschung im Bereich der Phytopharmakologie und des mittlerweile doch recht umfassenden klinischen Wirksamkeitsnachweises von pflanzlichen Arzneimitteln verkümmern.

Die Anwendung von Phytotherapie im Bereich der Pflege mit Wickeln, Auflagen, Einreibungen und Tees ist offensichtlich im akutmedizinischen Bereich besser zugänglich mit hoher Akzeptanz beim Pflegepersonal und verbunden mit einem starken Engagement. Ideal wäre die Kooperation beider Berufsgruppen mit einer Vernetzung phytotherapeutischer Anwendungen und der Verordnung von pflanzlichen Arzneimitteln als Reaktion auf die Bedürfnisse der Patientinnen und Patienten und im Sinne einer integrativen Grundhaltung gegenüber konventioneller und komplementärer Medizin.

\section{Dank}

Die Autorin dankt der SMGP (www.smgp.ch), insbesondere dem Geschäftsführer Prof. Dr. Beat Meier, gleichzeitig Dozent für Phytopharmazie an der Zürcher Hochschule für angewandte Wissenschaften (ZHAW), für die Begleitung des Projektes und die kritische Begutachtung dieser Publikation sowie des Lernskripts für das Pflegepersonal. Sie dankt weiterhin Marijke Frater, Ebnat-Kappel, Mitglied und Beauftragte der SMGP, für die Mithilfe bei der freiwilligen Prüfung.

\section{Literatur}

Bühring U: Freiburger Heilpflanzenblätter 1-4. Freiburg i.Br., Eigenverlag der Freiburger Heilpflanzenschule, 2002.

Bühring U: Praxis-Lehrbuch der modernen Heilpflanzenkunde. Stuttgart, Sonntag, 2005.

Bühring U, Sonn A: Heilpflanzen in der Pflege. Bern, Hans Huber, 2004.

Fischer-Rizzi S: Medizin der Erde. München, Heyne, 1999.

Jänicke C, Grünwald J, Brendler T: Handbuch Phytotherapie. Stuttgart, WVS, 2003.

Kalbermatten R, Kalbermatten H: Pflanzliche Urtinkturen. Baden, AT, 2005.

Oppliger P (Hrsg): Kräuterpfarrer Johann Künzle: Chrut und Uchrut. Aktualisierte und erweiterte Auflage. Baden, AT, 2008.

Reuter HD: Therapie mit Phytopharmaka. Ulm, Gustav Fischer, 1997.

Saller R, Reichling J, Hellenbrecht D: Phytotherapie, klinische, pharmakologische und pharmazeutische Grundlagen. Heidelberg, Haug, 1995.

Scherf G: Pflanzengeheimnisse aus alter Zeit. München, blv, 2004.

Scherf G: Die geheimnisvolle Welt der Zauberpflanzen und Hexenkräuter. München, blv, 2007.

Schilcher H, Kammerer S, Wegener T: Leitfaden Phytotherapie. München, Urban und Fischer, 2007.

Schönfelder P, Schönfelder I: Der KosmosHeilpflanzenführer. Stuttgart, Franck-Kosmos, 1995.

Sonn A: Wickel und Auflagen, Stuttgart, Thieme, 2004.

Stichmann W, Stichmann-Marny U: Der neue Kosmos Pflanzenführer. Stuttgart, FranckKosmos, 1999.

Storl W-D: Heilkräuter und Zauberpflanzen. Unveränderte Neuauflage von 1996. Aarau, AT, 2000.

Thüler M: Wohltuende Wickel. Worb, Maya Thüler, 1998.

Wiesenauer M: PhytoPraxis. Berlin, Springer, 2006.

Zimmermann W: Praktische Phytotherapie. Stuttgart, Sonntag, 1994. 